Recherches en histoire de l'art, histoire des civilisations, archéologie, anthropologie et muséologie

Cahiers 3

\title{
John Constable et le statut de l'esquisse
}

John Murdoch

\section{OpenEdition}

Journals

Édition électronique

URL : http://journals.openedition.org/cel/508

DOI : $10.4000 /$ cel.508

ISSN : 2262-208X

Éditeur

École du Louvre

Édition imprimée

Date de publication : 1 octobre 2013

Référence électronique

John Murdoch, « John Constable et le statut de l'esquisse », Les Cahiers de l'École du Louvre [En ligne], 3 | 2013, mis en ligne le 01 octobre 2013, consulté le 01 mai 2019. URL : http://

journals.openedition.org/cel/508; DOI : 10.4000/cel.508

\section{(c) $(1) \&$}

Les Cahiers de l'École du Louvre sont mis à disposition selon les termes de la licence Creative Commons Attribution - Pas d'Utilisation Commerciale - Pas de Modification 4.0 International. 


\section{Cahiers de l'École du Louvre recherches en histoire de l'art, histoire des civilisations archéologie, anthropologie et muséologie \\ Numéro 3. Octobre 2013}

\section{John Constable et le statut de l'esquisse John Murdoch}

Article disponible en ligne à l'adresse :

http://www.ecoledulouvre/revue/numero3octobre2013/Murdoch.pdf

Pour citer cet article :

John Murdoch, « John Constable et le statut de l'esquisse », Cahiers de l'École du Louvre, Recherches en histoire de l'art, histoire des civilisations, archéologie, anthropologie et muséologie [en ligne] n 3, octobre 2013, p. 44 à 52.

(c) École du Louvre

Cet article est mis à disposition selon les termes de la Licence Creative Commons Attribution - Pas d'utilisation commerciale - Pas de modification 3.0 non transposé. 


\section{Cahiers de l'École du Louvre recherches en histoire de l'art, histoire des civilisations archéologie, anthropologie et muséologie \\ Numéro 3. Octobre 2013}

\section{Sommaire}

\section{Éditorial}

Équipe de recherche

\section{Dossier: les revues}

Le « GR05 ». Approche de quelques revues du XX $\mathrm{XX}^{\mathrm{e}}$ siècle Introduction d'Hélène Klein

La présentation des objets africains dans DOCUMENTS (1929-1930), magazine illustré

Coline Bidault

Le conflit renaissant de la figure et de l'abstraction dans Labyrinthe, journal mensuel des Lettres et des Arts (octobre 1944-décembre 1946)

Blandine Delhaye

BROOM: An International Magazine of the Arts (1921-1924):

une revue d'avant-garde américaine

Ambre Gauthier

L'image de la guerre dans L'Élan (1915-1916), un refoulement apparent Hadrien Viraben

\section{Articles}

" John Constable et le statut de l'esquisse »

Conférence du 9 avril 2013 dans le cadre du séminaire doctoral

John Murdoch

«Adieu veau, vache, cochon, couvée... » La boucherie à l'Ancien Empire : croisement des données iconographiques, textuelles et archéologiques

Fanny Hamonic

L’entreprise patrimoniale de Louis-Joseph GUYOT (1836-1924)

à Dourdan, entre érudition et médiation

Léda Martines

p. $63-72$

\section{Actualité de la recherche}

Reconnaissance et médiation d'un patrimoine : vecteurs d'une identité territoriale? Journées d'étude de troisième cycle, 14 et 15 juin 2012

Noémie Couillard, Nicolas Navarro, Maylis Nouvellon p. $73-81$

Les « Ymagiers » à l'École du Louvre

Cycle de conférences organisées par l'IRHT 


\section{John Constable et le statut de l'esquisse}

\section{Conférence de John Murdoch}

Dans cet essai, je voudrais examiner les esquisses de l'artiste anglais John Constable, en exposant très sommairement le statut historique de l'esquisse dans l'art de la peinture européenne et dans la pratique de Constable, l'impact de son style « hérissé » sur sa réputation de son vivant, et l'effet produit par la redécouverte, après 1888, de l'ensemble de ses esquisses, sur sa fortune critique dès la fin du $\mathrm{XIX}^{\mathrm{e}}$ siècle (fig. 1).
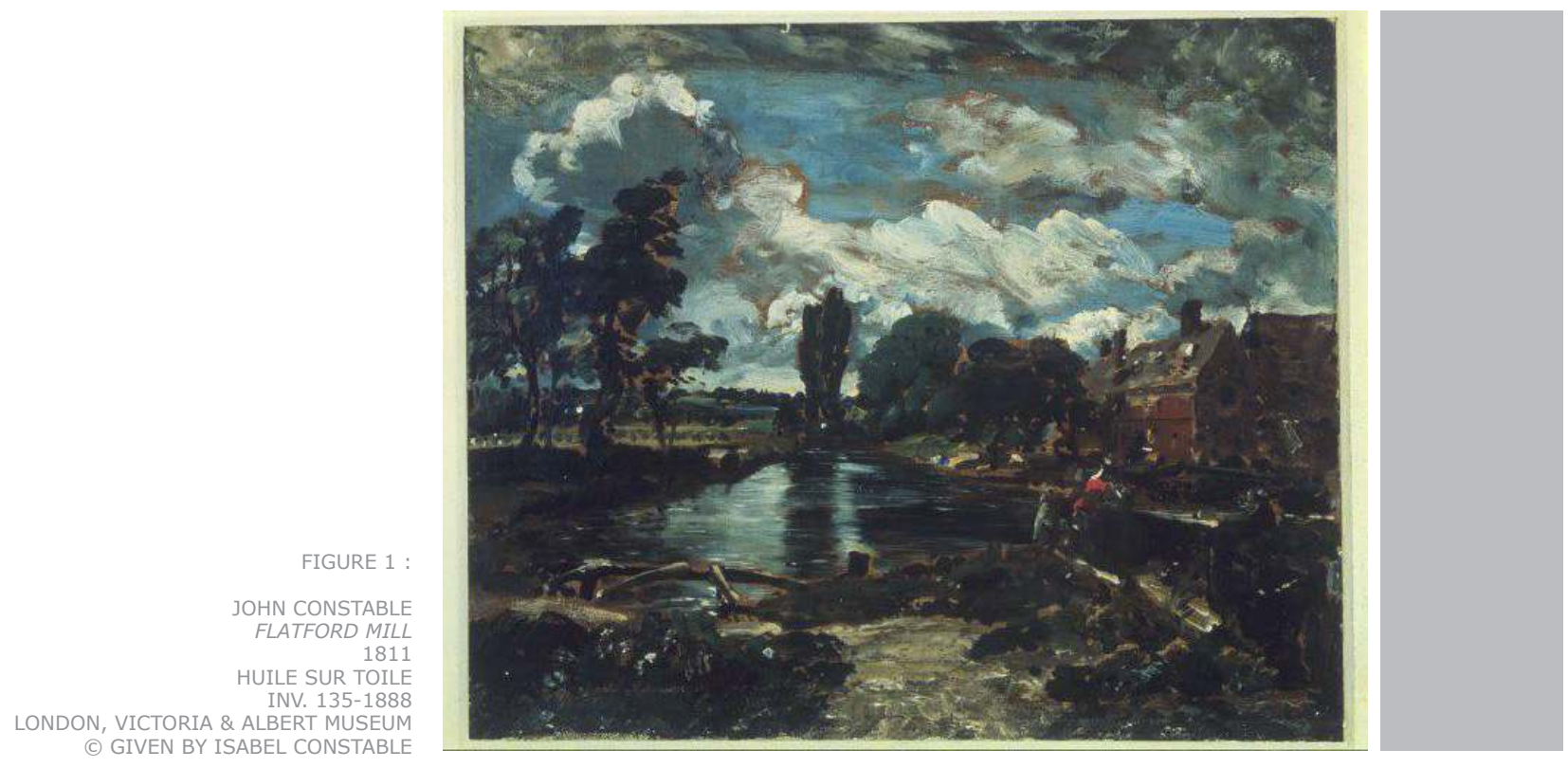

Constable, né en 1776, était l'héritier d'une tradition artistique qui, au moins depuis Rubens au début du XVII ${ }^{\mathrm{e}}$ siècle, attachait une importance spéciale à l'idée d'esquisse. L'esquisse semblait surtout le produit direct de la créativité de l'artiste. Elle représentait son génie, sa capacité à inventer des images, bien au-delà de celle des personnes ordinaires, et de les enregistrer alors qu'elles étaient encore fraîches dans son imagination. Au XVII ${ }^{e}$ siècle, les écrivains et critiques d'art regardaient Rubens, pour ses esquisses, comme le meilleur exemple de ce type de génie, décrivant comment « il restait parfois assis, les bras croisés, dans une extase d'énergie concentrée, et puis soudainement semblait se réveiller, tendait la main vers son pinceau, et faisait exploser le produit de son imagination échauffée dans une fureur de créativité sur un panneau devant lui » (Sanderson, 1657). Qu'il ait été capable de faire cela était dû à l'habileté suprême de son art, puisque les exigences techniques du dessin n'empêchaient point le déroulement de son activité intellectuelle. Les connaisseurs et collectionneurs accordaient ainsi la plus grande valeur aux esquisses de Rubens, à cause de leur évidente authenticité, voire leur capacité à représenter la personnalité, l'individualité et la puissance créatrice de l'artiste. Tout en reconnaissant que sa valeur dans l'histoire des nations civilisées était, sans aucun doute, due à ses grandes peintures finies, ils sentaient néanmoins que quelque chose de plus essentiel était clairement présent dans ses esquisses qu'elles étaient, dans un sens spécialisé, primaires, la véritable empreinte digitale de son génie. Dans leur matérialité, elles représentaient une sorte d'incarnation, un mystère à travers lequel la nature du génie pouvait être reconnue, et même possédée, par les gens du monde. 
Au XVIII ${ }^{e}$ siècle, cette idée, bien ancrée dans la culture des connaisseurs, finit par s'intégrer à ce que nous appelons le Romantisme. Dans les arts visuels, c'est la période où l'aquarelle atteint un statut autonome. Ce goût pour l'aquarelle est à mettre en parallèle, à cette époque et de plus en plus au cours du XIX ${ }^{\mathrm{e}}$ siècle avec l'intérêt artistique pour le paysage. Dans l'art anglais, nous remarquons les dessins des jeunes voyageurs, John Robert Cozens, Francis Towne, John Warwick Smith et Thomas Jones, durant les décennies 1760-1780. Avec la réouverture de l'accès à l'Europe du sud et à la Méditerranée en 1802, Turner et ses disciples ajoutèrent des images de la Suisse et de l'Italie au répertoire du Grand Tour. Par son caractère transportable et sa réalisation sur place, l'aquarelle arrivait à offrir une vision personnelle de l'apparence réelle des sites fameux de l'histoire et de la mythologie, et exerçait, de ce fait, un effet sur les imaginations sensibles.

Ce genre de paysage devenait aussi le support d'un autre aspect de l'imagination romantique, l'intérêt porté aux expériences qui ont modelé, soit dans son enfance, soit dans sa maturité, la véritable personnalité de l'artiste. C'est évident parmi les poètes, Byron et Wordsworth en particulier, ceux qui avaient rapporté dans leurs épopées autobiographiques, Childe Harold's Pilgrimage et The Prelude, leurs réactions émotives devant des lieux particuliers et leur impact sur la formation de leur conscience artistique. Vers 1803, plusieurs jeunes peintres ont également eu à cœur de préciser les circonstances par lesquelles ils avaient eu l'expérience de ces lieux, grâce aux particularités de la lumière, de l'heure ou du temps. Peu importe si ces lieux n’avaient pas de signification historique, ils atteignaient désormais une dimension majeure, puisqu'ils avaient saisi l'œil de l'artiste. Voilà Robert Hills, Cornelius Varley, Robert Havell, John Linnell, William Mulready faisant des esquisses en plein air à l'aquarelle ou grâce à cette technique, relativement transportable, de l'huile sur carton. Leurs sujets étaient des bâtiments indigènes, des fermes et les champs, les arbres, les mauvaises herbes au bord des rivières, les gravières, moulins et écluses. L'intérêt était précisément que ces sujets n'étaient ni recherchés ni composés, mais immédiats et locaux, isolés par l'œil de l'artiste et étudiés souvent avec un détail minutieux exigé par une signification aperçue soudainement. De telles études des gravières ou bords de chenaux n'étaient pas en outre inutiles dans le cadre des techniques de la peinture; ces esquisses, conformes à l'entraînement traditionnel de l'Académie et de l'atelier, constituaient un matériel brut pour produire une peinture composée et finie, à laquelle ces détails apportaient des éléments de fraîcheur et d'originalité. Comme Walter Scott l'évoquait, "peeping and botanizing » (entrevoyant et herborisant) sur les bords de la Greta en été 1812, il amassait des détails pour le poème Rokeby qui lui assureraient un accès à la variété inépuisable de la nature et ainsi, le moyen de lutter contre la banalité menaçant tous les arts classiques. Par cette voie, il espérait atteindre l'originalité. Pour les peintres aussi, une étude minutieuse et personnelle d'un lieu bien connu, précisément parce qu'il était proche et familier, pouvait ouvrir la porte à une cuvre plus fraîche et frappante, et être saluée par les critiques comme « originale » (fig. 2).

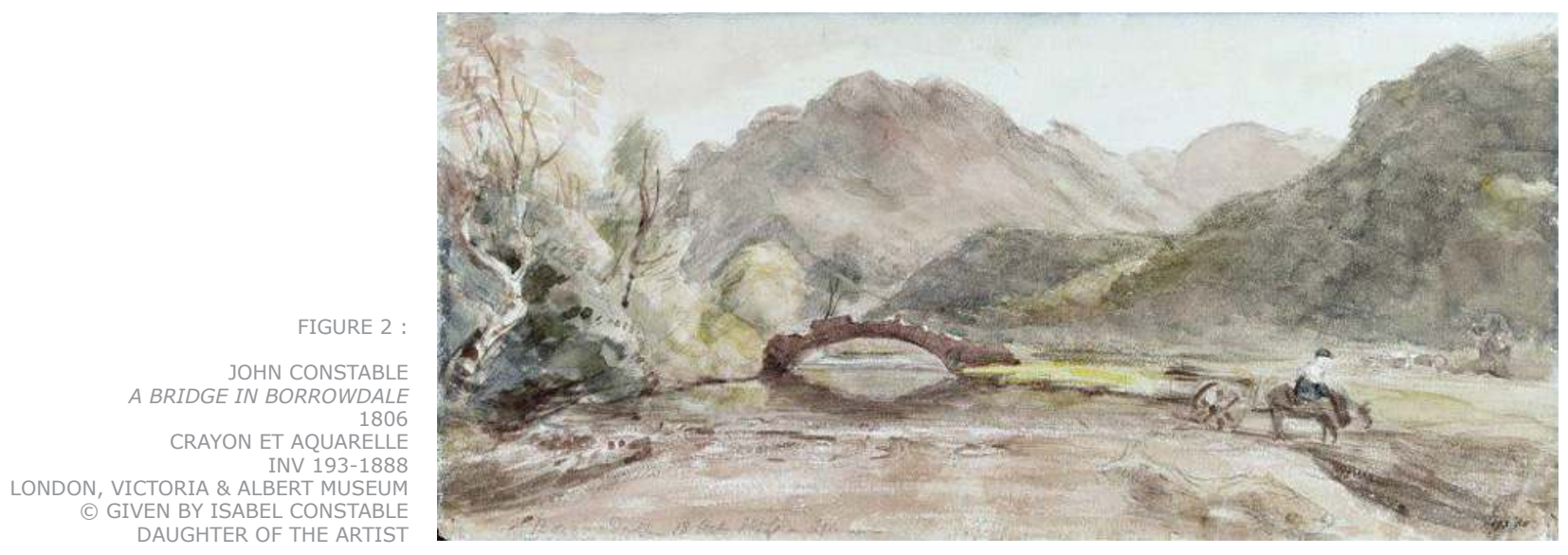


C'est ici, que nous retrouvons John Constable, à un moment très intéressant de son évolution artistique. En 1806, il avait fait un périple automnal dans le Lake District, où il avait été profondément ému par la pastorale vivante de cette région - par l'économie bergère de Borrowdale, son classicisme solennel à la manière de Poussin, les montagnes, les lacs et les petites villes grises d'alentour. Ses nombreuses esquisses à l'aquarelle en témoignent. Quelques vestiges de son travail à l'huile survivent aussi, mais rarement un panneau entier, parfois seulement des traces visibles aux rayons $\mathrm{X}$ sur une toile ou sur un panneau réutilisé les années suivantes. En 1807, 1808 et 1809, il a présenté à l'Académie Royale des sujets du Lake District, mais y a renoncé en 1810 ; par la suite, il semble avoir détruit toute trace de ce premier attachement à ce genre de sujet.

S'agissait-il d'une crise intime ou d'une décision raisonnable et stratégique ? J'opterais pour la seconde hypothèse, parce que c'est véritablement le moment où il tourne le dos à la mode classique de la pastorale - mode qui a dominé l'art du paysage dans la tradition académique - et adopte celle de la géorgique, genre qui, malgré une très large présence dans la poésie anglaise dès le XVII ${ }^{\mathrm{e}}$, avait été très peu utilisé par les peintres anglais, et presque jamais dans les expositions académiques. À la place des images traditionnelles des bergers innocents vivant librement des fruits de la terre dans une mise en scène naturellement noble, Constable introduisait le paysage d'après le péché, le paysage où les hommes doivent travailler - voire labourer (fig. 3) - le paysage des bornes et des clôtures, des maisons, de l'agriculture, des moissons et des moulins, des fleuves rendus navigables par les écluses, des bateaux et de la construction des bateaux - en bref, tout ce qu'avait évoqué Virgile dans sa première Géorgique pour caractériser la vie humaine dans «l'âge de fer» actuel. Nous pouvons dire qu'il avait abandonné le paysage touristique pour le paysage de son propre milieu économique, pour les scènes de son enfance et de sa jeunesse. En 1811 et les années suivantes, il a traversé et retraversé ce paysage familier comme un géomètre, étudiant la structure et la disposition des objets dans un paysage qui deviendrait pour lui, le fondement d'une carrière artistique. Il est facile de voir comment il a pu être attiré par cette orientation artistique vers ce qu'il connaissait absolument et personnellement, une orientation qui le rendait maître d'un genre classique, sans risquer la banalité de la pastorale. Avec la géorgique, il pouvait rester fidèle aux principes de grandiloquence classique qu'exige la sphère publique pour réaliser des tableaux finis qui puissent attirer l'attention et lui permettre d'obtenir l'admission dans les rangs de l'Académie - ce que désirait tellement le jeune Constable.

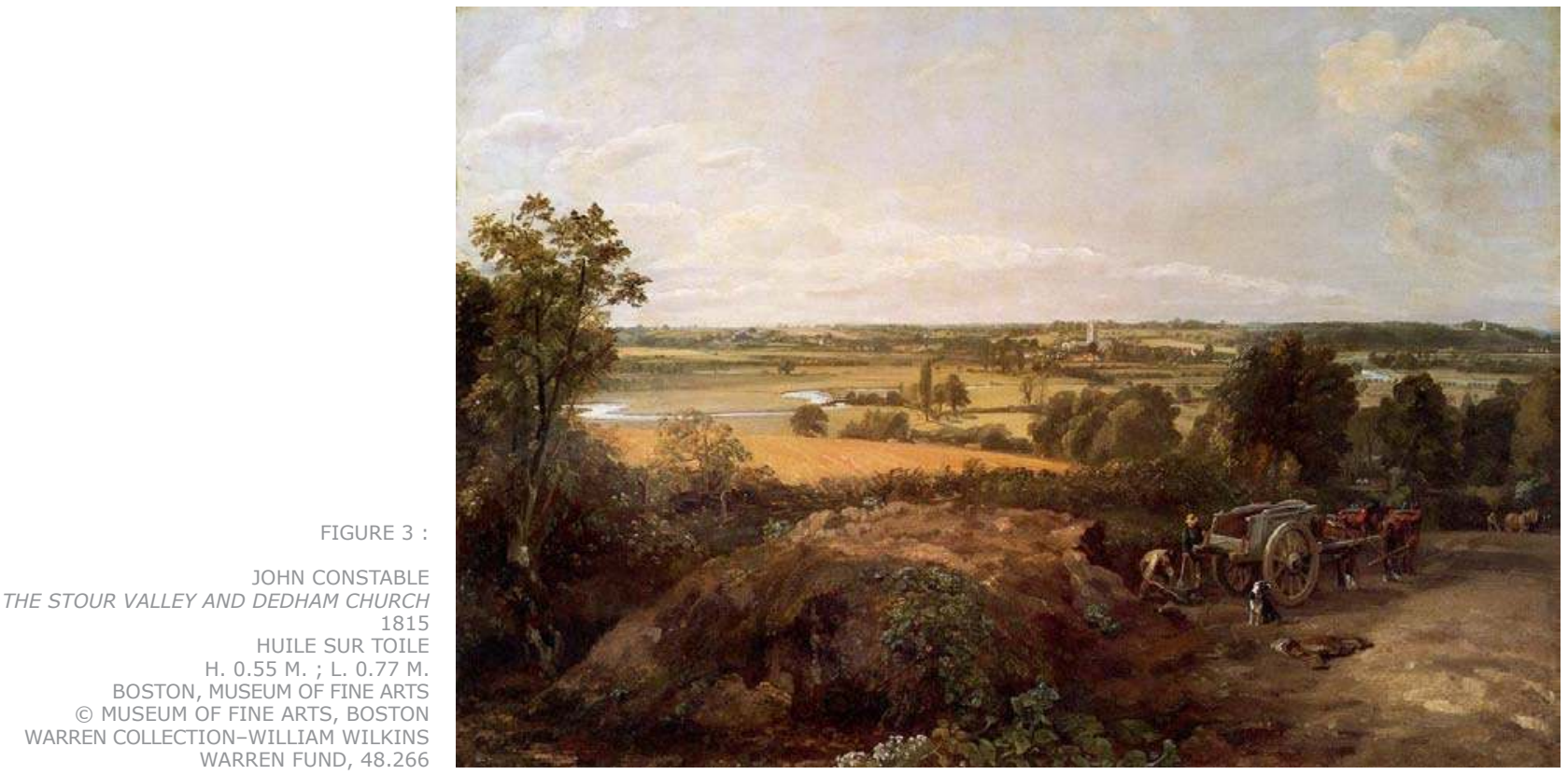


Les esquisses à l'huile d'après 1812 annoncent et forment une partie de sa production de peintures pour les expositions pendant les quinze années qui suivent. Leurs sujets sont des scènes typiques de l'agriculture et de l'industrie qui formaient la base de l'économie de la région d'East Anglia. Ces esquisses ont été le moyen - ou plutôt le témoignage des moyens - par lequel il a trouvé sa propre voie artistique, son authenticité et son originalité. Bien qu'exercices privés et professionnels, en rien destinées au public, elles ont laissé bien des traits sur les tableaux finis de cette période. Les critiques de Constable remarquaient déjà cette caractéristique : «Si la vigueur, la fraîcheur, et la vérité d'effet, apparentes dans tous les autres parties de l'œuvre, étaient associées à un peu plus de netteté dans l'exécution, la peinture serait parfaite. » (1822) (fig. 4 et 5).
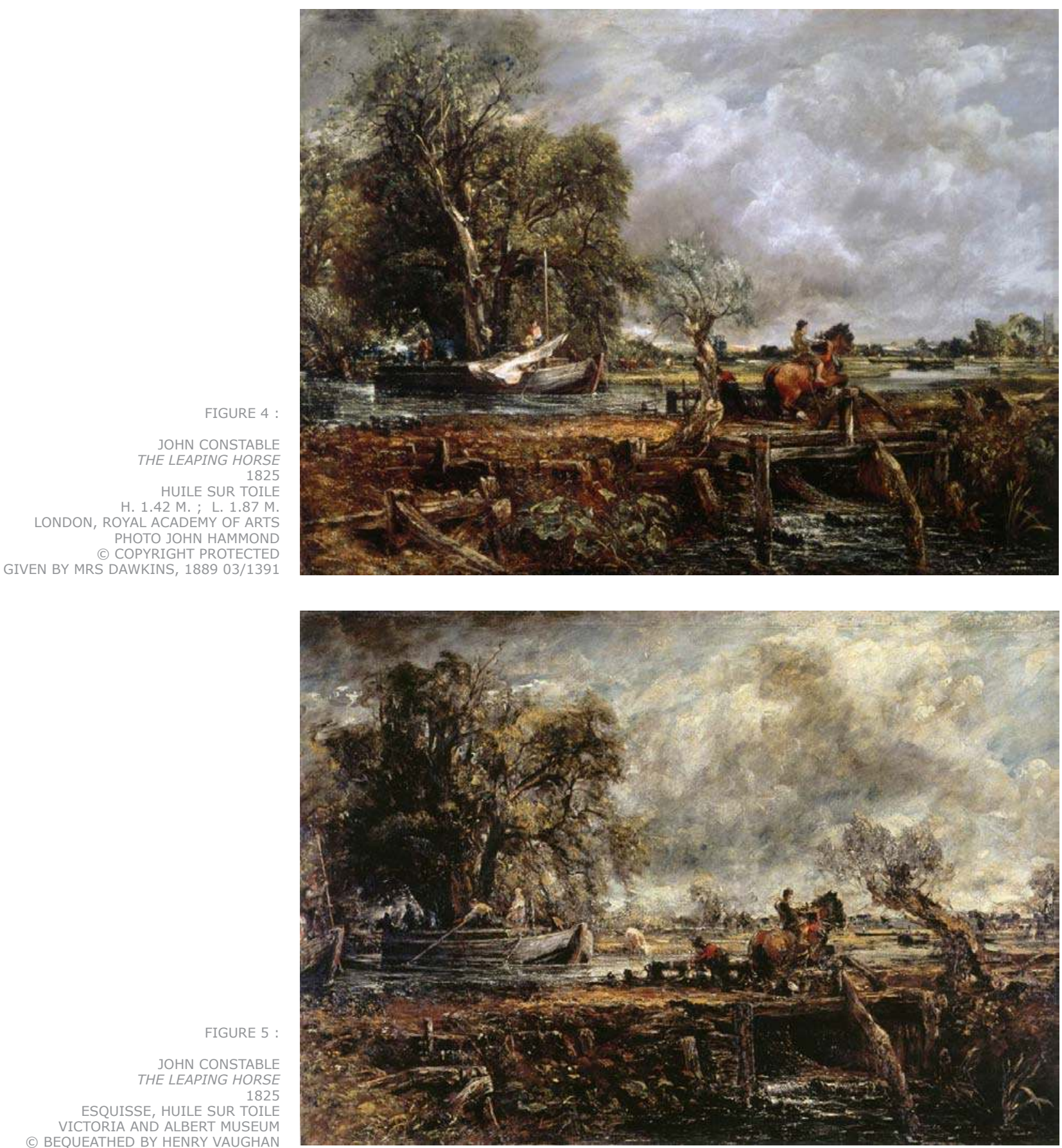
Pendant les années 1819-1825 Constable réalise la série extraordinaire de six paysages de six pieds sur des sujets géorgiques de l'East Anglia, The White Horse (1818-1819), Stratford Mill (1819-1820), The Hay Wain (1820-1821), View on the Stour (1821-1820), The Lock (1823-1824), The Leaping Horse (1824-1825), suivis par trois, Chain Pier, Brighton (1826-1827), Hadleigh Castle (1828-1829), Salisbury Cathedral (1829-1831) sur des sujets également engagés dans le monde actuel de l'«âge de fer».

Chacun donnait lieu à un tableau bien fini pour l'exposition et à une " esquisse» correspondante de même taille. Ces deux grands tableaux étaient le résultat d'une série d'esquisses nécessaires pour la mise en place des détails et de la composition, mais en principe ces esquisses restaient dans l'atelier et seul le tableau fini était offert au public. La totalité représentait sans doute un investissement énorme, en temps et en génie, et il est peu surprenant que la touche habituelle d'un artiste si profondément ancré dans la pratique de l'esquisse soit toujours sur le point d'apparaittre dans n'importe quelle partie d'une œuvre par ailleurs bien finie pour une présentation publique. Avec le succès de ses grands paysages, donc avec l'amélioration de ses perspectives personnelles et professionnelles, avec sa maitrise technique et sa rapidité d'exécution acquises grâce à des années de travail - oserat-on insinuer ici, avec l'arrogance même de Rubens ? - il semble avoir transféré de plus en plus franchement le caractère des esquisses dans les tableaux destinés à l'exposition. La qualité de l'immédiateté n'était plus réservée aux versions privées, mais se révélait dans les envois eux-mêmes. D'où le refus en 1829 de Pâturages à Salisbury : «Otez cette chose verte malpropre » ont dit les tapissiers de l'Académie qui ignoraient que c'était une œuvre d'un Académicien ayant le privilège de passer outre l'admission. Voilà comment « la touche » de l'esquisse est parfois devenue dominante dans les tableaux des années 1830 et d'où dérive la notion de «style tardif de Constable ».

Dans ses derniers tableaux, le style est assez extrême et aboutit à un manque de forme, un défaut de proportions, des perspectives fausses et une lumière composée presque entièrement de reflets troublants.

On peut dire, vraiment, que tout cela n'est probablement qu'une réponse au culte de la virtuosité qui a beaucoup enthousiasmé ses collègues académiciens dans les années 1830 - une réponse proche de celle des jeunes artistes fascinés par le spectacle de Turner peignant devant ses confrères lors du vernissage à l'Académie. Une facture aussi démontrée que démonstrative est devenue dans ces années un indice du charisme proprement dit de l'artiste, témoignant de sa capacité d'invention et d'exécution, précisément sans plus besoin des préparations élaborées et des études détaillées de la tradition académique. Mais les critiques, qui ont souvent réagi négativement devant le Turner des années 1830 et 1840, ont été aussi affectés par les couches de peinture inattendue chez Constable, de plus en plus troublées vers sa mort en 1837. Ainsi en 1835 l'un dit : « [Les sujets] sont habilement composés sur la toile. Mais, en les traitants, le peintre a conféré à son pinceau une licence extrême par rapport à sa propre manière extraordinaire et le résultat est moins agréable qu'il n'aurait pu être. Des blancs et des gris, des couleurs pâles, sont tamponnés avec profusion, comme s'ils étaient éclaboussés d'une brosse trop généreuse, ou sont hachurés et barbouillés à peine secs, si bien qu'à la fin l'effet des amas d'ombres en est affaibli. L'image entière acquiert, avec cette manière insolite de peindre, une apparence brute et tapissée...» Quand Constable décéda soudainement en 1837, la majeure partie de son œuvre restait invendue dans son atelier à Charlotte Street et chez lui à Hampstead. Bien qu'académicien et homme bien connu et respecté - ayant atteint, selon les contemporains, le deuxième rang du génie - Constable restait un artiste peu couronné de succès commercial.

Il est vrai qu'il a lui-même aggravé - encore influencé probablement par Turner - l'impression d'avoir tout gardé par devers lui, en rachetant, de temps en temps, un tableau à un client ou sur le marché. Après sa mort, il y eut dans la famille une tendance pieuse à conserver, autant que possible, la plus grande part de son œuvre. Malgré une vente majeure en 1838, vente imposée aux exécuteurs 
testamentaires pour assurer le devenir des orphelins, la plus grande partie de l'œuvre de l'artiste - comprenant presque la totalité de ses esquisses - continua à rester dans la famille pendant les cinquante années qui suivirent. Dans les années 1880, avec le décès des enfants de Constable, le fond d'atelier s'ouvre enfin au marché. Dans l'intervalle, le marché et le goût critique avaient beaucoup changé et continuaient à évoluer dans une direction très favorable à Constable.

Le climat critique européen du premier tiers du XIX ${ }^{e}$ siècle avait été dominé par des orthodoxies académiques, mises en place en Angleterre surtout dans les Discours de Joshua Reynolds. Ces doctrines proposaient pour les tableaux destinés aux espaces publics, un dessin aux effets larges et simples. Elles furent largement remises en cause au milieu du XIX siècle par la peinture allemande et par le Naturalisme défendu par les Préraphaélites et les doctrines de John Ruskin. Dans un tel contexte, la réputation de Constable ne pouvait que s'établir sur le tableau bien fini The Cornfield, acheté spécialement par un syndicat des amis de l'artiste pour qu'il figure au sein de la National Gallery. Celui-ci et d'autres tableaux finis, comme ceux acquis par le collectionneur John Sheepshanks et présentés au musée nouveau à South Kensington en 1857 (Salisbury Cathedral, Boat-Building, Flatford Mill, etc.), ou ceux qui furent exposés dans les grandes expositions du milieu du siècle, ont assuré à Constable une popularité fondée sur la conception qu'il était un patriote et avait donné à la nation sa propre image idéale. Cependant, dans les années 1880, le marché, les collectionneurs et surtout les critiques ont commencé à s'intéresser à la peinture de Barbizon, de La Haye et aux autres écoles contemporaines du continent. Les collectionneurs anglais, tels que Constantine Alexander Ionides et Chauncy Hare Townshend qui étaient en train de former des collections d'art moderne anglais, français, autrichien et suisse, estimaient le nouveau musée à South Kensington plus ouvert que la National Gallery, puisque celle-ci n'accueillait pas l'art moderne, ni d'ailleurs les esquisses, car sa mission était précisément de collectionner les œuvres majeures réalisées pour les espaces publics. Aussi, quand Isabel Constable, dernière survivante de la famille de l'artiste, a observé la situation, avant d'offrir sa part de l'héritage, elle choisit le musée de South Kensington. Sa donation en 1888 de quatre cent tableaux, esquisses et dessins est déterminante dans l'histoire de l'appréciation de l'œuvre de Constable. L'ampleur de son investissement dans l'art de l'esquisse était maintenant visible, et celles-ci, se trouvant à côté de la collection Townshend et bientôt de celle de Ionides, apparurent naturellement comme des modèles de l'art moderne. Tant qu'on en restait à reconnaittre l'influence de Constable sur les paysagistes français à partir du Salon de 1824, il était naturel de le considérer dans la perspective de la peinture française. Et de là il n'y avait qu'un très petit pas à faire pour voir Constable comme le critique allemand Julius Maier Graefe en 1908:

«Des choses comme cette petite Scène de côte (une esquisse à Brighton qui se trouvait dans une collection française) sont la première évidence de cette conception de la Nature que nous appelons l'impressionnisme, et préfigure les apports de Manet dans ce même domaine $»$.

Une étude, conservée au Victoria \& Albert Museum, de deux arbres s'inclinant à travers le plan de l'image, le motif brusquement coupé par la bordure supérieure, avec une figure féminine à l'arrière-plan, et esquissé en noir, est très fréquemment citée par les critiques à l'appui de cette thèse.

Dans Landmarks of Nineteenth Century Painting (1927), Clive Bell présentait Constable comme prophète de l'impressionnisme : bien que les esquisses soient «peut-être la partie de son œuvre la plus lumineuse et caractéristique, les tableaux finis eux-mêmes, en tant qu'ils incorporent la technique de l'esquisse, c'est-àdire les ouvrages tardifs, peuvent être admirés »; « on trouvera la qualité de ses tableaux, vus de près, souvent comme cassée, excitante, et moderne, comme celle d'un Renoir».

Quant à Roger Fry et ceux qui étaient influencé par son formalisme, au milieu du XX $X^{e}$ siècle, ils regardaient les tableaux les plus finis de Constable, y compris les grandes peintures de six pieds exposées à l'Académie et au Salon, comme représentatifs de tout ce qui était le plus mauvais dans l'art anglais : 
«L'élaboration de ces grosses machines-là, qui étaient calculées pour produire un effet dans les expositions de l'Académie... était entièrement mauvaise. Ils étaient presque toujours un compromis avec son idée réelle... Toutefois, heureusement, il a fréquemment fait des études à échelle réelle pour ces tableaux, et ce sont vers eux, et vers les esquisses, qu'il faut se tourner pour retrouver le vrai Constable. »

Cette posture critique domina jusqu'aux alentours des années 1970. Il est audelà de ma portée ici de suggérer une cause à l'abandon général de certaines orthodoxies dans ces années, mais je me souviens de quelques champs de bataille. Un de ceux-là fut la conférence associée à l'exposition Turner de 1975, à laquelle assistait le plus grand représentant de la tradition formaliste dans la critique artistique anglaise, le professeur Lawrence Gowing. Après le déjeuner, il nous donna une conférence, mais, passionné, il abandonna son texte pour se lancer dans une attaque contre les historiens de l'art britannique - personne n'était nommé - qui s'intéressaient chez Turner aux structures de narrativité et à l'iconographie de ses tableaux les plus fameux. Au premier rang de ceux-ci se trouvait John Gage, auteur de Colour in Turner (1969), suivi par les conservateurs et organisateurs de l'exposition en cours, qui pendant la conférence avaient déclaré leur intention d'élargir leurs études à la totalité de l'œuvre de Turner, y compris même sa poésie. Pour Gowing, c'était le pire des services possibles, une vraie honte, de s'écarter de cette manière de la célébration de l'entendement de la lumière, de la dissolution éclatante des formes chez Turner, pour chercher ce que ses peintures pouvaient bien vouloir dire - pire même, ce que l'artiste avait voulu dire, ou ce que les tableaux avaient signifié pour le public lors de leur exposition publique. L'art de la peinture, selon le professeur Gowing, ne voulait rien dire, ne devait rien signifier. Une peinture devait être seulement elle-même, une structure matérielle d'une beauté inhérente qui resterait naturellement immanente. Le travail du critique, et de l'historien de l'art (Gowing venait de prendre sa retraite à l'Université de Leeds), était de comprendre et de défendre ce fait, et en rien suggérer que la peinture puisse être au service de n'importe quoi d'autre. Assistant à cette conférence, disciple de John Gage, fasciné par sa révélation d’un Turner qui avait possédé une vraie personnalité, et une ambition de transmettre aux générations des sujets de grande importance, et une habilité suprême pour en faire, j’ai su que la voix de Gowing était celle d'un temps révolu.

L'année suivante, c'était le bicentenaire de la naissance de John Constable, et son tour de faire l'objet d'une exposition majeure. Je ne me souviens pas de confrontations publiques, mais l'exposition n'était pas moins remarquable comme la première présentation du travail fait par Leslie Parris et Ian Fleming Williams sur la topographie et l'iconographie des paysages de Constable. Ce travail éclaircissait la pratique des industries de l'Essex et du Suffolk durant la période de Constable, tels l'assolement, la construction et l'usage d'un monceau de fumier etc., clés pour l'esthétique de l'artiste comme pour la compréhension de son œuvre par le public. Dans les années suivantes, les points forts de l'érudition sur Constable demeurent les mêmes - des études iconographiques et sociologiques (Rosenthal, Hemingway), une étude sur «la découverte de Constable (FlemingWilliams et Paris) après son décès et une collection de critiques journalistiques publiées de son vivant et après sa mort (Judy Ivy), tout ce matériel concernant la dimension publique de son œuvre et sa capacité de communication avec les gens qui s'y sont intéressé de temps en temps. Naturellement, l'historiographie des années 1980 et 1990 a donné sa place aux esquisses, dans toute leur variété, mais pas, ou très peu, sous l'angle de la question du style ou de la facture. La restauratrice Sarah Cove a poursuivi au Victoria \& Albert Museum et à la Tate Gallery ses études très suggestives sur la construction et la facture de toutes les esquisses, mais les questions sur leur nature et leur destination ne sont vraiment revenues au premier plan qu'en 2006-2007. L'exposition Les Grands paysages de Constable de cette année, à Londres, Washington et San Marino, Californie, a rassemblé pour la première fois depuis la mort de l'artiste, tous les tableaux de six pieds, les peintures finies et les esquisses, y compris celles à l'échelle réelle - leurs doppelgangers - dans un contexte où il était possible de considérer l'étendue entière de sa pratique, de ses factures et de ses formats. 
L'exposition rassemblait ainsi presque tout ce qui peut faire avancer l'entendement de la pensée de Constable, quand il créait des esquisses à l'échelle réelle pour des peintures dont la composition était déjà bien planifiée. Comme toutes les questions les plus intéressantes, celle-ci n'a pas de réponse facile, et peut être même n'y en a-t-il aucune. Ainsi ce serait une approche un peu ingénue, par exemple, que de se demander si une grande esquisse faciliterait, mieux qu'une petite, la composition d'un grand tableau. Car une grande esquisse n'était point nécessaire, Constable étant parfaitement compétent dans l'art de l'agrandissement par la mise au carreau; d'ailleurs, avoir en même temps deux grands toiles sur des chevalets dans l'atelier aurait présenté beaucoup d'inconvénients. $\mathrm{Ne}$ s'agissait-il pas plus d'un besoin ou d'une volonté de faire des modifications tardives au contenu ou à la composition principale du tableau : l'examen des pentimenti (repentirs) sur les esquisses à même échelle et les peintures finies témoigne du fait que l'artiste était parfaitement content de retoucher les unes et les autres, voire de faire des changements assez radicaux, de telle façon que les deux versions paraissent posséder véritablement une existence individuelle. Il n'est même pas certain que les esquisses à même échelle aient une priorité chronologique - c'està-dire qu'elles aient forcément précédé les peintures finies. Par ironie, c'est en quelque sorte grâce à la critique formaliste que nous pouvons percevoir, presque sans effort, que Constable, pour des raisons liées à son identité romantique et à son admiration pour Rubens, a pu considérer l'esquisse comme plus forte, voire plus authentique, dans l'expression de l'idée essentielle d'un tableau. Il n'est sans doute pas inutile de préciser qu'il n'existe rien dans la correspondance de l'artiste, et tout à fait rien dans les commentaires de son biographe et ami, le peintre Leslie, qui touche à l'existence même de ces grandes esquisses. On peut deviner peutêtre que Constable ne connait aucun moyen de s'interroger sur un tel sujet, un sujet qui en toute probabilité entraînerait pour lui des références présomptueuses aux grands maitres de la peinture classique, et une vue de sa propre œuvre dans la perspective exaltée de l'histoire et de la critique de l'art. Même Turner ne parla jamais de lui-même en de tels termes. 
Référence électronique

John Murdoch, "John Constable et le statut de l'esquisse », Cahiers de l'École du Louvre. Recherches en histoire de l'art, histoire des civilisations, archéologie, anthropologie et muséologie [en ligne] n ${ }^{\circ} 3$, octobre 2013.

URL: http://www.ecoledulouvre.fr/revue/numero3octobre2013/Murdoch.pdf

\section{cc)creative \\ $\circledast \ominus \circledast$}

\section{L'auteur}

John Murdoch, formé à Oxford, où il a réalisé son MPhil sur Walter Scott and the Picturesque, a occupé divers postes dans le domaine de la conservation au Royaume-Uni et aux États-Unis : conservateur de la Birmingham City Art Gallery, conservateur au département des peintures du Victoria \& Albert Museum de Londres, directeur-adjoint des collections du Victoria \& Albert Museum de Londres, il fut ensuite directeur de la Courtauld Institute Gallery de Londres et enfin directeur des Huntington Art Collections.

Il a assuré le commissariat scientifique de nombreuses expositions, notamment «Impressionism for Britain » (1994), «Frank Dobson, Sculptor» (1995), « William Chambers» (1996), « Roger Fry » (1999), and «Art on the Line » (2001), "The Beauty of Life: William Morris and the Art of Design » (2003-2004); " An Eye for Beauty: Collectors and the History of BritishWatercolor » (2004), "Sensation and Sensibility: Viewing Gainsborough's 'Cottage Door' » (2006), "Constable's Great Landscapes: The Six-Foot Paintings » (2006), « Pressed in Time: American Prints 1905-1950» (2007-2008).

John Murdoch est aussi l'auteur de nombreux ouvrages, parmi lesquels : Scott, Pictures and Painters (1971); George Eliot and Pre-Raphaelite Realism (1973); Byron (1974); British Watercolours (1977); David Cox (1981); The Discovery of the Lake District (1984); A Northern Arcadia (1985); Painters and the Derby China Workes (1987); The Landscape of Labor: Transformations of the Georgic (1990); The Courtauld Family and its Money (1994); Seventeenth-Century Portrait Miniatures in the Collection of the Victoria \& Albert Museum (1997); Architecture and Experience in Somerset House (2001); et Connoisseurship of Watercolor (2004).

John Murdoch est le premier chercheur invité par l'École du Louvre.

Pendant son séjour parisien, de février à avril 2013, il a poursuivi une recherche sur Somerset House, son influence sur l'architecture française et plus largement sur les échanges artistiques entre Londres et Paris à la fin du XVIII ${ }^{e}$ siècle.

Cette opération a pu être réalisée grâce au mécénat de la Fondation Daniel et Nina Carasso qui permet à un chercheur confirmé de séjourner à Paris deux ou trois mois pour mener à bien un projet scientifique sur lequel il a été sélectionné, tout en participant à la vie de la recherche à l'École, notamment aux séminaires de $3^{e}$ cycle et à une ou plusieurs conférences publiques. 